ISAHP Article: Strategic Supplier Selection: The Case of International Food Trading Company To Be Submitted to the International Symposium of the Analytic Hierarchy Process 2020, Web Conference.

\title{
Strategic Supplier Selection: The Case of International Food Trading Company
}

\author{
Chuvej Chansa-ngavej* and Natchanok Paramaporn \\ Regional Center for Manufacturing Systems Engineering, Chulalongkorn University \\ *Corresponding author: chuvej@gmail.com
}

\begin{abstract}
The objective of this research paper is to develop a strategic supplier selection system for an international food trading company using the Analytic Hierarchy Process (AHP). The products involved are canned food for worldwide export. The decision makers consist of the president and two marketing managers. The chosen decision criteria listed by weight of importance are "Export Capability" (54\%), "Pricing Policy" (28\%), "Technological Catch-up" (10\%), "Ethical Practices" (5\%), and "Environmental Management" (3\%). The sub-criteria chosen are "Number of Food Safety Certificates" (54\%), "Net Selling Price" (23\%), "Automation in Production Process" (8\%), "Payment Terms" (5\%), "Workers' Safety and Health" (4\%), "Supply Continuity" (2\%), "Packaging Varieties" (2\%), "Eradication of Child and Forced Labor" (1\%), and "Environmental Compliances" (1\%). According to the company's existing choice of suppliers using cheap price as the selection criterion, Supplier C is chosen for pineapple products, Supplier F for sweet corn products, and Supplier L for baby corn products. With the proposed strategic supplier selection system, the optimal suppliers for pineapple products are found to be B (with a score of 33.6\%) and D (32.8\%), suppliers for sweet corn products are F (29.4\%) and E $(29.2 \%)$, and for baby corn products are I (32.3\%) and K (26.6\%). Strategic criteria related to current business environments are found to receive substantial weights from our experts' opinion. The present work thus provides a case to substantiate the significance of strategic supplier selection and reinforces the notion that this important topic deserves a lot more attention from the business sector.
\end{abstract}

Keywords: strategic supplier selection, supply chain management, multi-criteria.

\section{Introduction}

The success or failure of the procurement function depends on the company's buying decision. The supplier selection stage benefits the company in terms of minimizing negative risk-taking from trading with an undependable supplier who warrants a replacement by top-notch performance service providers. The proper way to diminish the risk of suffering from mistakes is to find the right suppliers who provide reasonable price with great performance. However, the purchasing authority more-often-than-not purchases commodities from the lowest price supplier which in turn brings about a long list of troubles and supply chain disruption. Such problems directly affect corporate creditability in the eyes of business partners. In recent years, research publications on supplier selection have turned focus on "sustainable supplier selection". Many academics have been writing research articles about finding the imperative criteria to enhance the selection process sustainability. However, the hitch is that sustainable suppliers are not always the best performance suppliers. More research works on strategic supplier International Symposium on the 
ISAHP Article: A Style Guide for Paper Proposals To Be Submitted to the International Symposium on the Analytic Hierarchy Process 2020, Web Conference.

selection are needed to find the eligible potential suppliers by integrating efficient supplier qualifications, supplier risk management, and sustainable supplier selection as three major lenses for exploring relevant criteria. Criteria from past studies in the literature have mainly considered the economic and environmental themes, whereas social-theme criteria have often been overlooked although they seem to be more and more important in recent years. Also, human rights have become a hot topic, especially with regards to the call for elimination of unethical practices of legitimate workers as well as forced labors. Besides, technological innovations have emerged as a key role in reducing excessive cost, improving production efficiency, and strengthening positive image. When it comes to the economic dimension, the strategic supplier selection genre is much more sophisticated than the traditional supplier selection one. Numerous economic criteria apart from low-price quotation have been advocated such as quality, warranty, delivery cost, and payment terms. For the above reasons, the objective of the present work is to fulfill the gap of strategic supplier selection through the case application of an international food trading company which has three main products: pineapple, sweet corn, and baby corn canned food.

\section{Literature Review}

Supply chain efficiency could be improved by finding qualified suppliers. Candidate selection criteria should include risk and sustainability considerations. Risk is a factor to avoid various uncertainties such as natural disasters and supply disruptions (Alikhani et al., 2019). Optimal strategic supplier models in the literature comprise of Efficient Supplier Qualification (ESQ), Sustainable Supplier Selection (SSS), and Supplier Risk Management (SRM). Factors such as cost and quality are the fundamental factors, supply continuity is one of the useful factors in the food industry (especially vegetable and fruit industry) because crop growth is dependent on weather condition and natural disaster can damage the plant. Technological capabilities are another interesting element since innovation is an essential part of enhancing product quality and increasing product capacity. Environmental management focuses on ISO 14000 which is used to indicate that the supplier complies with environmental regulations and standards.

Zimmer et al., (2015) reviews 143 publications and decomposes the criteria relating to sustainable supplier management into three dimensions; economic, environmental, and social issues and three different levels in the hierarchical structure are dimension, main theme, and theme. Analysis of the themes revealed that the most used theme is economic with $52.5 \%$, followed by environmental $(38.1 \%)$ and social $(9.4 \%)$. The rare use of social criteria is perhaps due to the particularity of social issues and the difficulty in measuring and quantifying social sustainability. This area needs to be practically integrated with strategic supplier selection in the same way as the environmental theme. It is surprising that 'water' and 'energy' are not often mentioned amongst environmental criteria even though they are recommended by the United Nations (UN) and the Global Reporting Initiative (GRI). At the same time, 'Child and forced labor', 'Discrimination', and 'Abuse of human rights' often recommended by UN and International Labor Organization (ILO) are not listed at the top ten social criteria.

Lau et al. (2020) reviews the assessment of organic food suppliers and finds the top five primary criteria to be the cost of monitoring, certified organic and safety, quality, delivery, and product, in that order. For the fresh and organic food products, laboratory testing and non-organic producer from 'Cost of monitoring' are considered as crucial factors and 'Delivery' criteria is another significant factor since fresh and organic food products require lots of mandatory requirements such as refrigerated storage and need

International Symposium on the Analytic Hierarchy Process
2

WEB CONFERENCE

DEC. 3 - DEC. 6,2020 
ISAHP Article: A Style Guide for Paper Proposals To Be Submitted to the International Symposium on the Analytic Hierarchy Process 2020, Web Conference.

fast delivery to maintain freshness and reduce time to be contaminated by ambient environments. On the other hand, 'Quality', 'Certified product and safety', and 'Product' are fundamental and essential features for the entire food product categories.

In this paper, the selection of decision criteria would follow the principle adopted by Chansa-ngavej and Srijuntub (2010) in relation to the AHP method, which comprises of five elements: completeness, non-redundancy, decomposability, operationality, and minimum number of criteria.

\section{Hypotheses/Objectives}

The present research work aims to develop an AHP framework for the strategic supplier selection of an international food-product trading company, based on an adaptation of three strategic themes: ESQ, SRM and SSS.

\section{Research Design/Methodology}

\subsection{The strategic supplier dimensions}

ESQ, SSS, and SRM as the usually adopted strategic supplier selection dimensions are shown in Figure 1. They boil down to the three dimensions of Economic, Social, and Environmental as noted by Zimmer et al. (2015). In line with the current digital era, the

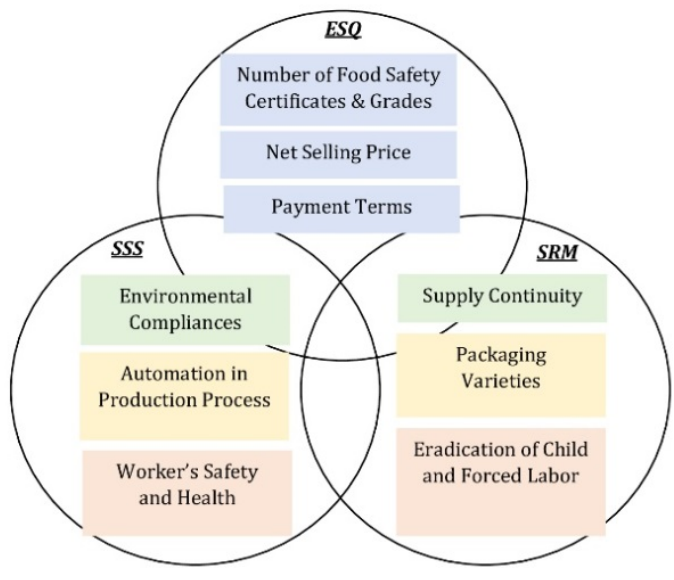

Figure 1 The usual classification of strategic supplier selection criteria present research work adds the 'Technological' dimension as the fourth dimension. To explain, cutting-edge technology may aid the company in locating more competitively priced suppliers. Food product consumers can notice a drop in standard from using cutprice materials. To be more economical, the supplier needs to leverage automated apparatus and utilize such valuable technologies to enhance food process and packages, including productivity growth, precise measurements, and cost saving.

4.2 Identify the "must" criteria for evaluation

"Must" criteria are the basic screening tool to identify the qualified potential suppliers. In the case company, one such "must" criterion is that the operational process and supplied products need to comply with food safety standards, specifically GMP and HACCP. Besides, potential suppliers must be capable of supplying two specified can sizes. Suppliers who lack any of the mandatory attributes will be disqualified.

4.3 Identify "want" criteria for strategic supplier evaluation

The key "want" criteria are selected from a prioritized set of success criteria combined with critical factors in the food industry and are classified by the usual dimensions in four hierarchical levels as shown in Figure 2.

4.4 Determine expert respondents and backgrounds

The company president and the marketing managers are chosen as the decision makers to judge the relative importance of the strategic supplier selection criteria. Other managers in the finance, shipping, and label and document functions are excluded due to their relative lack of experience in foreign grocery markets and specific requirements. 
ISAHP Article: A Style Guide for Paper Proposals To Be Submitted to the International Symposium on the Analytic Hierarchy Process 2020, Web Conference.

\section{Data/Model Analysis}

\subsection{Criteria and Sub-criteria Weights}

The priority weight of criteria and sub-criteria are shown in Figure 2. The rankings of the criteria are C1 (54\%), C2 (28\%), C5 (10\%), C3 (5\%), and C4 (3\%). Noticeably, the top two criteria are from the economic theme, making up $82 \%$. The technological criterion, $\mathrm{C} 5$, is trending at $10 \%$. The least proportion belongs to $\mathrm{C} 3(5 \%)$ and $\mathrm{C} 4(3 \%)$. Together, the total of these two criteria is less than C5 alone. The C.R. of the main criteria weighting is $8.4 \%(<15 \%)$, which is acceptable according to Saaty (1980).

Among the sub-criteria, C1.1 comes first, accounted for 54\%. As the case company's main products are canned food, all customers worldwide mandate the guarantee of sanitation and harmlessness. C2.1 wins against $\mathrm{C} 2.2$ by $18 \%$ because price can attract sale volumes and payment term can improve cash flow. C3 is a severe concern amongst the public. However, as management of a medium-sized company, our experts' opinion appears to deviate from the public norm, perhaps because only large companies tend to keep an eye on human workforces in order to boost public image. C3.1 is given 1\%, while C3.2 receives greater scores at $4 \%$. Ethical audits play a role in the judgement of illegal employment and security. Once the factory has no guarantees, goods cannot be exported to ethical distributors. End-buyers rarely asked for environmental compliances prior to making purchasing since natural resource protection is considered a fundamental responsibility. Further, the sum of $\mathrm{C} 4.1$ and $\mathrm{C} 4.2$ makes up 3\%. Continuity can sustain or

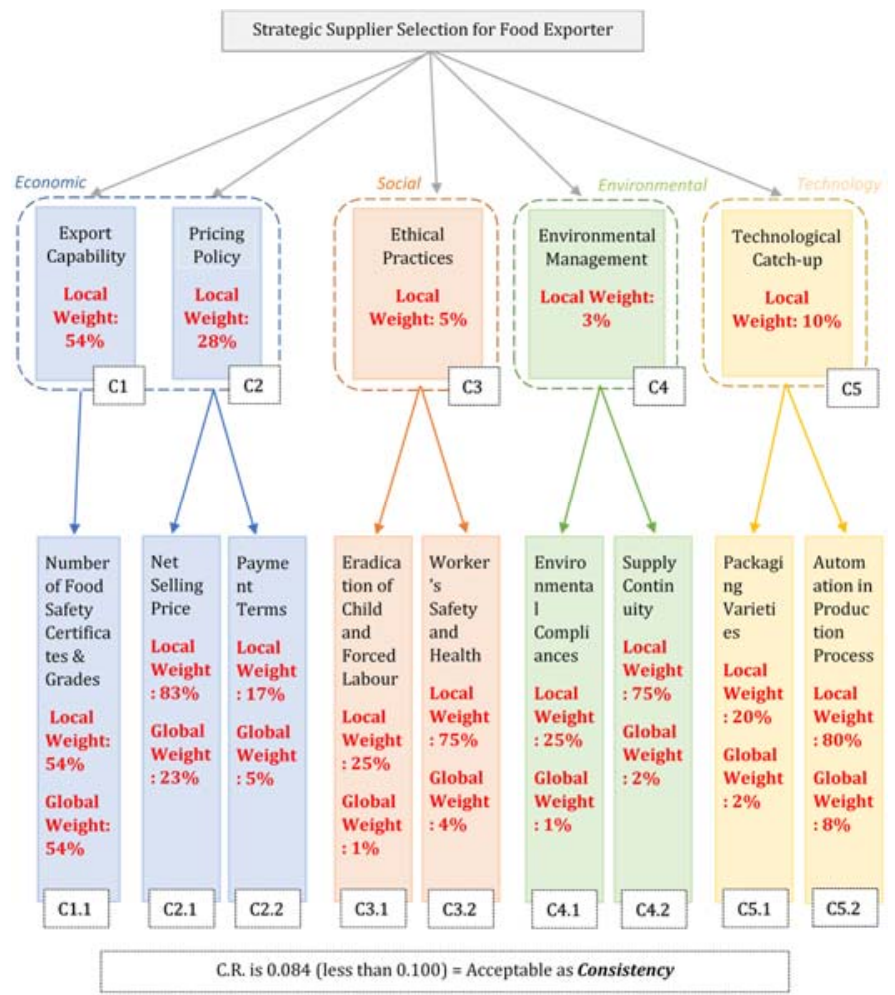

Figure 2 Priority weights of the supplier selection criteria ruin company's reputation, whereas distributors no longer ask for ISO inspection now. On account of technology which is now-a-days embedded in everything, C5 gain unexpected attention. The majority vote for C5.2, accounted for $8 \%$ out of $10 \%$. Automated manufacturing process means lower contamination and higher quality which impacts endcustomer perception. Packaging diversities, C5.1, help trade with fewer cluster of suppliers due to the ability to provide numerous categories filled with full container load, thus aiding in cost-cutting.

5.2 Final results of supplier selection

computing the scores of all the qualified pineapple suppliers, the first rank belongs to Supplier B, followed closely by D. Suppliers occupying the next places which should be listed by the case company as backup suppliers are A and C, in that order. For the sweet corn suppliers, the first place belongs to F, followed by E. The reserved vendors are $\mathrm{H}$

International Symposium on the Analytic Hierarchy Process 
ISAHP Article: A Style Guide for Paper Proposals To Be Submitted to the International Symposium on the Analytic Hierarchy Process 2020, Web Conference.

and $\mathrm{G}$, in that order. The first place for baby corn supplier's performance belongs to I, followed closely by $\mathrm{K}$. The reserved vendors are $\mathrm{L}$ and J respectively.

\section{Limitations}

The dimensions of the factors considered for strategic supplier selection could be further studied to understand if risk and sustainability for each individual supplier should be included as part of business environments. The criteria established in the present research are for canned vegetable and fruit export community which might not be the proper criteria for other types of business and need further adjustment.

\section{Conclusions}

Applying strategic supplier selection will benefit the company by promoting procurement success, compared to the traditional supplier selection criteria of Quality, Cost, and Delivery. In recent years, considering only such traditional criteria is insufficient because there are so many complex considerations of concern to a particular type of business. However, cost and quality are still vital for food export industry as our experts them the top two weight scores. For the years ahead, the "red ocean" era - where competing is mainly on price - will disappear. The advent of the "blue ocean" era - where creating new market space will become the norm - will replace the old style of competition. The traditional criteria will be of less concern and the modern strategic criteria will gain more attention in place of the conservative ones.

Despite the relatively low weight scores of SRM and SSS criteria, there is still the tendency for the expert's mindset to change toward giving more scores to those strategic criteria due to a myriad of reasons that could bring a long list of problems. As an example, the recent supermarket ban in the UK on Thai coconuts because of the accusations by PETA that Thai coconuts are harvested by abused monkeys. It follows that ethical certificates to certify non-abusive labors would become essential overnight. In these days, consumers are keenly aware of sustainable products selection prior to making purchases, contributing to increase the weight of environmental factors. ISO 14000 is the beginning step to help decrease environmental impacts to production process. Thailand's economy heavily relies on agricultural products in her role as the world's leading food exporter. Production competency should be another vital factor to surge productivity yield in response to high consumption demands. Our food export experts assign a moderate weight of importance to the technological dimension since supply disruptions

take place in some seasons. Providing that more advanced technology is adopted, the trading company can supply food products to foreign distributors without a gap month.

\section{Key References}

Alikhani, R., Torabi, S. \& Altay, N., 2019. Strategic supplier selection under sustainability and risk criteria. International Journal of Production Economics, 208, 6982.

Chansa-ngavej, C. \& Srijuntub, A., 2010. Using Analytic Hierarchy Process for innovative technology selection: a case study. International Journal of Innovation and Learning, 8(3), 279.

Lau, H., Shum, P., Nakandala, D., Fan, Y. \& Lee, C. (2020). A game theoretic decision model for organic food supplier evaluation in the global supply chains. Journal of Cleaner Production, 242, 118536.

Zimmer, K., Fröhling, M. \& Schultmann, F. (2015). Sustainable supplier management - a review of models supporting sustainable supplier selection, monitoring and development. International Journal of Production Research, 54(5), 1412-1442.

International Symposium on the Analytic Hierarchy Process 